\title{
Study on Dynamic Guidance District Division Inside the Parking Lot
}

\author{
Shi Yanyana, Han Ying \\ Qingdao Binhai University, Qingdao 266555, Shandong Province, China \\ a471618585@qq.com, b37382430@qq.com
}

\begin{abstract}
Keywords: Dynamic guidance, Partition Model, Simulated annealing algorithm
Abstract. According to the division principle and method of parking inducing community, a parking induction partition model is established to determine the objective function and constraints of the study. The simulated annealing algorithm is used to study the partition model. According to the principle of simulated annealing method, the calculation process of the optimal solution of parking induced partition model is established. Finally, a simple numerical example is given, the optimal solution calculated demand, which are three of the Pareto optimal solution for analysis and comparison, the results show that the rationality of the model and algorithm.
\end{abstract}

\section{Introduction}

Dynamic guidance district division inside the parking lot is the basic content of parking guidance system. It is one of the key issues to better organize parking behavior. Whether the Dynamic guidance district division inside the parking lot is reasonable or not, can directly affect the rationality of berths allocated to vehicles and the parking efficiency of the entire parking lot.

\section{The Building of Partition Model}

1) The determination of the objective function. In order to turn the actual interior parking lot into a mathematical model problem, now the parking lot internal road structure is simplified to a standard $m \times n$ matrix plot $G_{i j}$.some of the plots may be combined into a regular matrix plot. A plot that can not be reduced to a matrix can add virtual plots to form a matrix plot. the virtual plot parameters set to zero.

2) The determination of constraints. According to the car park guidance district division principle, two of the important principles are translated into objective functions and the rest are transformed into constraints. Each matrix plot can only belong to one induced plot.

(1) In order to ensure the range of the plot information bearing for each parking, the number of matrix cells included in each parking-inducing plot is set to be greater than or equal to two.

(2) In order to avoid too many or too few berths in the parking guidance district set the number of berths for each parking-inducing plot as:

(3) Due to the different interior layout of the parking lot and the size of the parking lot. In order to simplify the calculation, define the size of the induced plot through the side length.

(4)Induced plots are divided into rectangular rules as far as possible. Any induced plot can not be cut off by other induced plots, So as to making the display screen to get the best use and provide the driver with the complete surrounding guidance information.

\section{The Application of Simulated Annealing Algorithm in the Model}

The application of simulated annealing algorithm in combinatorial optimization can be summarized as follows: First assume that each solution to a combinatorial optimization problem corresponds to a microscopic state. The objective function of combinatorial optimization is set to f(i) which corresponds to a micro-state E(i). The temperature of the annealing process is converted 
into control parameters. Then calculate from the initial solution and control parameters. Loop iterative process of generate new solution-calculate difference of objective function-accept / discard. With the gradual attenuation of control parameters, the algorithm iterates continuously until the current solution obtained when the algorithm terminates is the approximate solution to the optimal solution.

1) The methods to generate new solutions. After the initial solution is determined, the new solution is randomly generated according to any one of the following two methods:

(1) $G_{i j k}=1, G_{i^{\prime} j^{\prime} k^{\prime}}=1$ converted to $G_{i j k^{\prime}}=1, G_{i^{\prime} j^{\prime} k^{\prime}}=1 . k, k^{\prime}$ is the sequence of Induce plot.

(2) The original formula is from $G_{i j k}=1$ into $G_{i j k}=0$. The original formulais from $G_{i j k^{\prime}}=0$ into $G_{i j k^{\prime}}=1$.

2) The acceptance criteria of new solution. Through the concept of Pareto solution and the Metropolis criterion, we judge the accepting conditions of new solution:

Comparing the new solution and the old, the new solution is accepted when the new solution prevails over the current solution. Otherwise, a random number $\xi$ of $[0,1)$ intervals should be generated by the random number generator. If $r$ is greater than this random number, then consider the state of the new solution important to accept the new solution, otherwise discarded. This method of treatment can accept the disadvantages solution so as to avoid the calculation into a local optimum.

3) The initial temperature selection. The choice of the initial temperature is important for the entire calculation. The following two points should be noted:

(1) Initial temperature $T_{0}$ should be large enough. if the value is too small can easily lead to the algorithm into the local optimum and can not get the optimal solution.

(2) The initial temperature should not be chosen too large. if the value is too large can easily lead to an increase in the number of iterations of the algorithm, reducing the efficiency of the algorithm.

The initial temperature is generally selected by the following formula:

$P_{0}=\exp \left(-\frac{C_{\max }-C_{\min }}{T_{0}}\right)$

$P_{0}$ - A value close to 1 but less than 1. $C_{\max }$ - The maximum value of the objective function.

$C_{\min }$ - The minimum value of the objective function.

The initial temperature should also be selected according to the application of the simulation results adjusted.

4) The cooling strategy of temperature control parameters. The global search performance of the simulated annealing algorithm is directly related to the annealing speed. In the same state to conduct a comprehensive search for the optimal solution is the most favorable. But it is necessary to consider the increase of the time cost caused by the comprehensive search. Therefore it is important to control the annealing speed. That is set the appropriate temperature control parameters cooling strategy. Usually there are four temperature control parameters cooling strategy, they are log cooling, rapid cooling, linear cooling and exponential cooling. In order to simplify the calculation of the index choose the cooling index strategy.

$$
T_{\text {new }}=a \times T_{\text {current }}
$$

$T_{\text {new }}$-Updated temperature. $T_{\text {current }}$-Current Temperature.

$a$-Cooling parameters.(Generally take $0.80 \sim 0.98$ )

5) The calculation of termination condition. The algorithm's process can simply be described as performing a random search of states at each temperature as the temperature decreases. When the temperature drops very low, the optimal solution is obtained with probability 1 .Thus the algorithm consists of two cycles. The first layer is the inner loop that performs a random search at the same temperature. The second layer is the outer loop that is the cooling process and the 
conditions for the final termination.

The terminated conditional of program calculation are usually taken as the number of iterations that have been set or the number of consecutive new solutions that have not been accepted or the temperature has expired. Take a fixed number of steps to terminate the condition. That is taking a fixed number of steps sampling under each temperature condition. After a fixed number of steps to carry out the temperature of the outer loop. The outer loop is accepted as no termination condition in successive cycles.

6) The selection of initial solution. The initial solution should be given before the simulated annealing calculation. It is the starting point for the algorithm to begin iteration. The initial solution should be chosen so that the algorithm leads to a better final solution, The selection of initial solution leads to a better final solution for the final algorithm. A large number of experimental results show that Simulated annealing is a "robust" algorithm. The final solution to the algorithm does not depend very much on the choice of initial solution. Therefore, the initial solution can be randomly selected.

7) The steps of simulated annealing algorithm are as follows: Step1: Randomly give the initial value $G_{i j k}^{0}$ of induced plot division in the parking lot. According to the initial value calculate the objective function value $Z_{1}^{0}$ and $Z_{2}^{0}$. According to cooling strategy calculating the initial temperature $T_{0}$, Setting the number of iterations of the outside loop is $\mathrm{K}$ and setting the fixed step length of the inner loop is $K_{2}$.

Step2: Calculate the number of matrix plots which expressed with the symbol $Y_{k}$ that do not meet the constraint conditions in each induced plot by the limitation of the constraint conditions. In the current state the selection of the matrix cell $G_{i j}$ is performed according to $P(k)$ which is the probability chosen to produce a new solution. Its essence is to select the induced cells that do not meet the constraints of the number of matrix cells and more. The expression of $P(k)$ is as follows:

$$
P(k)=\frac{1+Y_{k}}{k+\sum_{k=1}^{k} Y_{k}}
$$

Step 3: The matrix cell selected in step 2 is iteratively calculated to obtain a new solution $G_{i j k}^{\prime}$, and then then the computer randomly chooses any of the two methods to generate new solutions to find the new solution.

Step 4: Verify the new solution in the two matrix cells whether satisfy all the constraints? If they are satisfied continue the calculation. Otherwise, return to step 3 to regenerate the new solution.

Step 5: Calculate the objective function value of the new solution $G_{i j k}^{\prime}$ corresponds to the objective function $Z_{1}$ and $Z_{2}$, and choose the new solution according to the aforementioned new solution acceptance criterion.

Step 6: It is detected whether the number of cycles of the inner cycle has exceeded the fixed value $K_{2}$ of the fixed step. If satisfied, proceed to the next calculation. Otherwise, return to step 2 to continue the cycle.

Step 7: Judge the solution by the definition of Pareto optimal solution. If it satisfies the definition of Pareto optimal solution, it will be liberated into Pareto solution set filter. if not satisfied it do not put in Pareto solution filter. If the filter is full, then according to the principle of the niche technology for the superfluous kicked out.

Step 8: Determine whether the number of outer cycles exceeds a given number of cycles $K_{1}$.if not, cool according to the cooling strategy $T_{n e w}=a \times T_{\text {current }}$. and then return to step 2 to continue the cycle. if the number of cycles is satisfied $K_{1}$, the optimal solution is obtained and the solution is output. 


\section{The Analysis of an Example}

Assume an ideal layout of the internal layout of the parking lot which is 200 meters long and width. The total area is 40,000 square meters. Each matrix cell has a horizontal and vertical length of 40 meters. As shown in Figure1. The figure indicates the number of berths that each matrix cell can provide and the number of berths required for one of its peak periods.

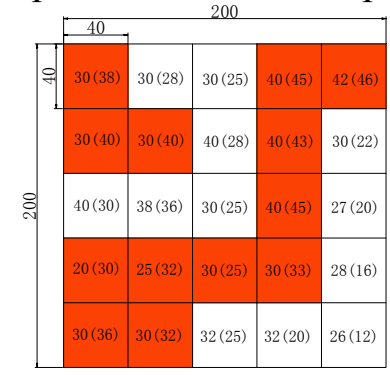

Fig. 1 The layout Figure of Parking interior

In Figure 1, the shaded matrix cell indicates that the probability of being parked is usually high and the traffic around it is relatively crowded. The matrix is as follows.

$$
C_{i j}=\left[\begin{array}{ccccc}
1 & -1 & -1 & 1 & 1 \\
1 & 1 & -1 & 1 & -1 \\
-1 & -1 & -1 & 1 & -1 \\
1 & 1 & 1 & 1 & -1 \\
1 & 1 & -1 & -1 & -1
\end{array}\right] S_{i j}=\left[\begin{array}{ccccc}
30 & 30 & 30 & 40 & 42 \\
30 & 30 & 40 & 40 & 25 \\
40 & 42 & 42 & 40 & 25 \\
20 & 25 & 30 & 30 & 28 \\
25 & 30 & 30 & 30 & 26
\end{array}\right] D_{i j}=\left[\begin{array}{ccccc}
35 & 28 & 25 & 45 & 45 \\
40 & 40 & 20 & 40 & 22 \\
30 & 36 & 26 & 45 & 20 \\
30 & 32 & 28 & 32 & 16 \\
26 & 26 & 20 & 18 & 14
\end{array}\right]
$$

After obtaining the above data, the simulated annealing calculation is performed on the example. First, set the initial value of the parameter.

The initial temperature is $T_{0}=10$. The annealing coefficient is $\mathrm{a}=0.95$. The number of fixed steps $K_{2}$ is 20 . Pareto solution filter capacity is 10 . The number of plots is $d=5$. the parameters are substituted into the model, then 10 Pareto optimal solutions are finally obtained through calculation. Through analysis and comparison, three Pareto optimal solutions are selected for explanation. The solutions to these three solutions and their respective objective function values are shown in the following figure.
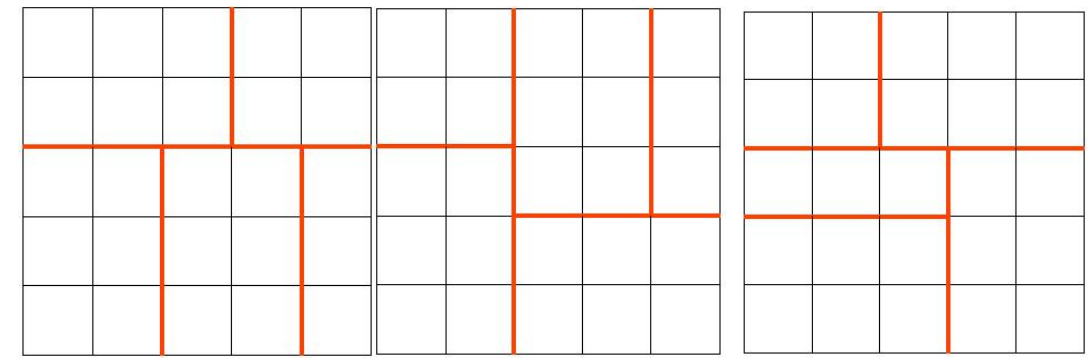

Program $1 Z_{1}=110, Z_{2}=7 ;$ Program $2 Z_{1}=106, Z_{2}=7$

Program $3 Z_{1}=106, Z_{2}=11$

Fig. 2 The Figure of Three Pareto optimal solution

The three solutions listed in Fig.2 are all Pareto optimal solutions. They dominate each other. They are all non-inferior solutions corresponding to the cell division. So these three options are all options. This problem is a multi-objective combinatorial optimization problem, and many non-inferior solutions are usually obtained in the process of solving multi-objective combinatorial optimization problems. The decision maker screens the resulting plan through a multi-criteria decision making approach. Eventually the policy makers get the most satisfied program as the final plan of parking interior induced plot division. 


\section{References}

[1] DUAN Man-zhen, CAO Hui-yun: Parking Lots Distribution Model for the Individual Demand Vol. 16 (2016), p. 153-159

[2] YUAN Jing: Improved Ant Colony for Solving the Optimal Parking Space Problems Vol. 41 (2013), p.376-377

[3] WEN Ya: Key Laboratory of Road and Traffic Engineering of the Ministry of Education Vol. 43 (2007), p. 215-216

[4] ZHANG Bao-yu,ZHOU Xiang-ting:Urban Parking Guidance System Planning and Design Induced Partition Classification Determination Vol. 12 (2007), p. 69-71

[5] CUI Yan-ping,TANG Ban-min:Research on the Strategy and Content of China Intelligent Transportation Development Strategy Vol.3 (2), p.28-31

[6] Information on http://www.doc88.com/p-2466355777853.html 